\title{
Optimisation-based parameter estimation of photovoltaic modules
}

\section{Mohamed A. Awadallah* and Bala Venkatesh}

\author{
Centre for Urban Energy, \\ Ryerson University, \\ 350 Victoria Street, Toronto, ON M5B 2K3, Canada \\ Email: awadalla@ryerson.ca \\ Email: bala@ryerson.ca \\ *Corresponding author
}

\begin{abstract}
This paper presents a methodology for parameter estimation of photovoltaic (PV) modules based on global search and optimisation algorithms. A nonlinear optimisation problem is formulated to search the best set of equivalent circuit parameters which minimises a certain objective function. The objective function measures the discrepancy between computed and targeted performance. The proposed technique is flexible on the source of the targeted performance, which could be datasheet information or experimental measurements. The method is also flexible on the nature and number of sought parameters according to the equivalent circuit representation and available information. The nonlinear optimisation problem is solved using three different global search routines, namely, genetic algorithms (GA), particle swarm optimisation (PSO) and bacterial foraging (BF). Effectiveness of the proposed methodology is shown through three case studies on different PV modules of various ratings and manufacturers. The extracted parameters could accurately simulate the performance of PV modules under normal operation as well as low irradiance and partial shading conditions.
\end{abstract}

Keywords: photovoltaic modules; parameter estimation; optimisation.

Reference to this paper should be made as follows: Awadallah, M.A. and Venkatesh, B. (2018) 'Optimisation-based parameter estimation of photovoltaic modules', Int. J. Industrial Electronics and Drives, Vol. 4, No. 1, pp.33-43.

Biographical notes: Mohamed A. Awadallah received his $\mathrm{PhD}$ from the Department of Electrical and Computer Engineering at the Kansas State University, USA in 2004. He is currently a Visiting Research Fellow with the Centre for Urban Energy at the Ryerson University, Toronto, ON, Canada and a Professor of Electrical Engineering with the Zagazig University, Egypt (on leave to Ryerson University). His research interests include motor drives, energy storage and renewable energy. He is a member of the Eta Kappa Nu, Tau Beta Pi and Phi Kappa Phi. He is a Registered Professional Engineer in the Province of Ontario, Canada.

Bala Venkatesh received his $\mathrm{PhD}$ in Electrical Engineering from the Anna University, Chennai, India in 2000. He is a Professor and Academic Director of the Centre for Urban Energy at the Ryerson University, Toronto, ON, Canada. His research interests include power system analysis and optimisation. He is a Registered Professional Engineer in the Provinces of Ontario and New Brunswick, Canada.

\section{Introduction}

Classical power sources depending on fossil fuels emit greenhouse gases and add to the world's global warming and environmental pollution problems. Renewable energies, which have recently received increasing research attention, provide green, free and sustainable sources. Motivated by governmental incentives, solar photovoltaic (PV) panels are rapidly penetrating electrical power systems worldwide. Large solar farms can produce power in several megawatts, whereas small residential rooftop installations have ratings in the range of few kilowatts. In both cases, accurate modelling is imperative for performance computation, operation scheme design and maximum power point tracking (MPPT) control.

Precise estimation of the equivalent circuit parameters is inevitable for analytical modelling of any electrical system or equipment. The PV module is no exception. Typically, equivalent circuit parameters of many electrical systems are computed based on the experimental results of prototype laboratory testing. Nevertheless, some publications available in the literature consider extracting PV module parameters from datasheet information supplied by the manufacturer. Analytical methods are proposed to express equivalent circuit parameters in terms of performance characteristics of the PV module (Farivar and Asaei, 2011; Chatterjee et al., 2011; Lo Brano et al., 2010; 
Ahmad et al., 2012; Cubas et al., 2013; Siddique et al., 2013; Sera et al., 2007; Ishaque et al., 2011; Chenni et al., 2007; Chan and Phang, 1987; Maoucha et al., 2012; Phang et al., 1984; Jia et al., 1995; Ortiz-Conde et al., 2006; de Blas et al., 2002; Bouzidi et al., 2007; Chan et al., 1986; Hejri et al., 2016). Accordingly, equivalent circuit parameters are estimated if module performance is known under some operating conditions with some simplifying assumptions. Such performance could be obtained from the manufacturer supplied datasheet of the module (Farivar and Asaei, 2011; Chatterjee et al., 2011; Lo Brano et al., 2010; Ahmad et al., 2012; Cubas et al., 2013; Siddique et al., 2013; Sera et al., 2007; Ishaque et al., 2011; Chenni et al., 2007) or from experimental measurements (Chenni et al., 2007; Chan and Phang, 1987; Maoucha et al., 2012; Phang et al., 1984; Jia et al., 1995; Ortiz-Conde et al., 2006; de Blas et al., 2002; Bouzidi et al., 2007; Chan et al., 1986). The mathematical expressions of module parameters are usually nonlinear and require iterative numerical solutions (Chatterjee et al., 2011; Lo Brano et al., 2010; Siddique et al., 2013; Sera et al., 2007; Ishaque et al., 2011; Chenni et al., 2007; Chan and Phang, 1987; Maoucha et al., 2012; Ortiz-Conde et al., 2006; Bouzidi et al., 2007); however, certain assumptions can enable the separation of variables and allow direct solution to identify the parameters (Farivar and Asaei, 2011; Ahmad et al., 2012; Cubas et al., 2013; Phang et al., 1984; Jia et al., 1995; de Blas et al., 2002; Chan et al., 1986). Although most of the work reported in literature considers the single-diode model of PV cells, a few publications are concerned with parameter estimation of the double-diode equivalent circuit (Ishaque et al., 2011; Chan and Phang, 1987; Maoucha et al., 2012). In Hejri et al. (2016), open-circuit voltage, short-circuit current and voltage and current at maximum power point are used to provide an initial point that guarantees convergence of analytical estimation routine. The parameters of a single-diode five-parameter model are accurately obtained based on analytical approximation of the model equations.

Another method of parameter estimation of PV modules depends on fitting measured and computed performance with minimal error (Chan et al., 1986; Hejri et al., 2016; Ikegami et al., 2001; Haouari-Merbah et al., 2005; Chegaar et al., 2006). Module performance is measured in the laboratory and then model parameters that get computed characteristics as close as possible to measurements are computed. Mathematical techniques of curve fitting vary between least-square method over the whole operating range (Ikegami et al., 2001), voltage fitting near opencircuit and current fitting near short-circuit (HaouariMerbah et al., 2005) and auxiliary function fitting (Chegaar et al., 2006).

Biologically-inspired optimisation algorithms are exploited to estimate PV module parameters via minimising certain objective functions (Ye et al., 2009; Sandrolini et al., 2010; Zagrouba et al., 2010; Krishnakumar et al., 2013; Ishaque and Salam, 2011; da Costa et al., 2010; Yeh et al., 2017; Oliva et al., 2017; Awadallah and Venkatesh, 2016; Awadallah, 2016; Chin et al., 2017). The targeted performance is obtained either through measurements (Ye et al., 2009; Sandrolini et al., 2010; Zagrouba et al., 2010) or through datasheet information (Krishnakumar et al., 2013; Ishaque and Salam, 2011; da Costa et al., 2010). Particle swarm optimisation (PSO), genetic algorithms (GA), bacterial foraging (BF), artificial immune systems (AIS), and differential evolution (DE) routines are used to estimate parameters of the single- and double-diode models. In Ye et al. (2009), the discrepancy between measured and computed module current is used as an objective function to be minimised by PSO and GA. Statistical and cluster analyses are used with PSO to fit measured performance to that computed through the seven-parameter double-diode model of PV modules (Sandrolini et al., 2010). The difference between measured and computed current is minimised via GA to extract PV module parameters (Zagrouba et al., 2010). In Krishnakumar et al. (2013), an objective function based on the rate of change of module current with respect to its voltage, at maximum power point, is minimised using BF, GA, and AIS. Information about maximum power point is obtained from the manufacturer datasheet. A similar objective function is minimised by DE in Ishaque and Salam (2011). Mean square error comparing computed current and that supplied by the manufacturer datasheet is minimised by DE to estimate the single-diode model parameters (da Costa et al., 2010). An improved simplified PSO algorithm is employed to extract the parameters of both single- and double-diode models of PV cells (Yeh et al., 2017). In Oliva et al. (2017), the chaotic whale optimisation algorithm is used to estimate the mathematical model parameters of solar cells. The internal parameters of the optimisation algorithm are dynamically updated as iteration progresses, which help improve convergence speed and accuracy.

Synergy between different optimisation algorithms can also enhance the performance; a PSO-guided BF routine can outperform both PSO and GA in extracting PV model parameters (Awadallah and Venkatesh, 2016). In addition, variations of algorithm parameters impact the convergence behaviour such that experimental numerical runs may be required before the best combination is reached. In Awadallah (2016), various combinations of BF algorithm parameters are experimented before the best fit for PV parameter estimation problems is realised. On the other hand, analytical and optimisation methods could be combined yielding a hybrid parameter estimation routine which can improve convergence behaviour and modelling accuracy (Chin et al., 2017). Moreover, an innovative estimation technique based on the learning, nonlinear mapping, and pattern recognition capabilities of artificial intelligence tools is presented in Salem and Awadallah (2014). Intelligent modules based on artificial neural networks and adaptive neuro-fuzzy inference systems are trained using data generated by the mathematical model to identify the best parameter set of PV cells.

This paper presents a technique based on optimisation routines to estimate the equivalent-circuit parameters of $\mathrm{PV}$ modules. The optimisation algorithm minimises an 
objective function expressing the relative absolute error (RAE) between targeted and computed performance. The targeted performance could be obtained through the datasheet supplied by the manufacturer or through experimental measurements. When datasheet information is used, targeted performance represents the open-circuit voltage, short-circuit current, maximum power and voltage at maximum power, all under standard test conditions (STC). On the other hand, open-circuit voltages and shortcircuit currents at different values of solar irradiance and cell temperature are used as targeted performance obtained via prototype laboratory testing. Three optimisation algorithms are used to solve the present problem, namely, GA, PSO and BF. The technique is tested on three PV modules with different ratings and manufacturers. The viability is verified by the good matching between measured performance and that computed through the estimated parameters. Extracted parameters could accurately represent system performance under normal operation as well as low irradiance and partial shading conditions.

The proposed technique of PV parameter estimation has various advantages not attributed altogether to any of the methods available in the literature. Firstly, the targeted performance spans over the whole range of operation from open-circuit to short-circuit points. Consequently, the obtained parameters are more representative of module behaviour under different conditions. Normally, the usage of datasheet characteristics at STC eliminates the need to perform prototype laboratory testing. However, for aged PV modules, whose performance deteriorates from that of the datasheet or in case datasheet is not available, experimental testing would yield more realistic parameters. Secondly, the proposed method avoids complicated mathematical derivations, inaccurate iterative solutions to nonlinear equations and misleading approximating assumptions. In addition, information on the derivatives of the objective function is not needed. Thirdly, the objective function formulation is simple, straightforward and meaningful where a wide variety of optimisation algorithms can be employed. Fourthly, the proposed technique can estimate a selected set of parameters of the single- or double-diode models of PV modules based on information availability and user preference.

\section{PV modelling and problem statement}

As shown in Figure 1(a), a PV cell can be modelled with a current source in parallel to a diode, a shunt resistance to account for leakage current and a series resistance to represent losses related to load current (Farivar and Asaei, 2011; Chatterjee et al., 2011; Lo Brano et al., 2010; Ahmad et al., 2012; Cubas et al., 2013; Siddique et al., 2013). Accordingly, the cell current is given as

$$
I_{c}=I_{p h}-I_{o s}\left[e^{\frac{q}{A K T}\left(V_{c}+R_{s} I_{c}\right)}-1\right]-\frac{V_{c}+R_{s} I_{c}}{R_{s h}}
$$

where $I_{c}$ is the cell current, A, $I_{p h}$ is the photocurrent, A, $I_{o s}$ is the reverse saturation current of the diode, $\mathrm{A}, q$ is the electron charge, $\mathrm{C}, A$ is the diode ideality factor, $K$ is Boltzmann constant, $\mathrm{J} /{ }^{\circ} \mathrm{K}, T$ is the cell temperature, ${ }^{\circ} \mathrm{K}, V_{c}$ is the cell voltage, $\mathrm{V}, R_{s}$ is the series resistance, Ohm and $R_{s h}$ is the shunt resistance, Ohm. The photocurrent depends on the solar irradiance and cell temperature and is given as

$$
I_{p h}=\lambda\left[I_{n}+k_{i}\left(T-T_{r}\right)\right]
$$

where $\lambda$ is the solar irradiance, $\mathrm{kW} / \mathrm{m}^{2}, I_{n}$ is the nominal short-circuit current at STC $\left(1000 \mathrm{~W} / \mathrm{m}^{2}\right.$ irradiance, $25{ }^{\circ} \mathrm{C}$ temperature and 1.5 air mass), $k_{i}$ is the short-circuit current temperature coefficient, $\mathrm{A} /{ }^{\circ} \mathrm{K}$ and $T_{r}$ is the reference temperature, ${ }^{\circ} \mathrm{K}$. Meanwhile, the reverse saturation current of the diode varies with temperature and is given as

$$
I_{o s}=I_{o r}\left[\frac{T}{T_{r}}\right]^{3} e^{\frac{q E_{g}}{A K}\left(\frac{1}{T_{r}}-\frac{1}{T}\right)}
$$

where $I_{o r}$ is the reverse saturation current of the diode at reference temperature and irradiance, $\mathrm{A}, E_{g}$ is the band gap energy of the cell material, $\mathrm{J} / \mathrm{C}$. When PV cells are connected in series and parallel forming a PV array in order to raise the level of output voltage and current, Figure 1(b), the output current is expressed as

$$
I=N_{p} I_{p h}-N_{p} I_{o s}\left[e^{\frac{q}{A K T}\left(\frac{V}{N_{s}}+\frac{R_{s} I}{N_{p}}\right)}-1\right]-\frac{1}{R_{s h}}\left[\frac{N_{p}}{N_{s}} V+R_{s} I\right]
$$

where $N_{p}$ and $N_{s}$ are the number of cells in parallel and series, respectively.

Equations (1) through (3) describe the performance of a PV cell. The model correlates the output variables of the cell, $V_{c}$ and $I_{c}$ with the independent variables representing the environmental conditions, $\lambda$ and $\mathrm{T}$, through physical constants and system parameters. The Boltzmann constant $(\mathrm{K})$, electron charge (q), band gap energy of the cell material $\left(\mathrm{E}_{\mathrm{g}}\right)$ and reference temperature $\left(\mathrm{T}_{\mathrm{r}}\right)$ are all constants. Whereas, series resistance $\left(R_{s}\right)$, shunt resistance $\left(\mathrm{R}_{\mathrm{sh}}\right)$, diode ideality factor $(\mathrm{A})$, nominal short-circuit current $\left(\mathrm{I}_{\mathrm{n}}\right)$, reverse saturation current at reference temperature and irradiance $\left(\mathrm{I}_{\mathrm{or}}\right)$ and short-circuit current temperature coefficient $\left(\mathrm{k}_{\mathrm{i}}\right)$ are parameters of the PV cell. Numbers of series and parallel cells are also required to compute the performance of a PV array using equation (4).

In the double-diode model of a PV cell, two diodes are in parallel with the photocurrent source as shown in Figure 2. The model is known to be more accurate than the single-diode model, especially at low irradiance levels. One diode represents the diffusion current in the $\mathrm{p}-\mathrm{n}$ junction, whereas the other takes the space-charge recombination effect into account. Both single- and double-diode models are widely accepted to represent the behaviour of crystalline semiconductor PV cells. The output I-V characteristic equation of the double-diode model becomes 


$$
\begin{aligned}
I_{c} & =I_{p h}-I_{o s 1}\left[e^{\frac{q}{A_{1} K T}\left(V_{c}+R_{s} I_{c}\right)}-1\right] \\
& -I_{o s 2}\left[e^{\frac{q}{A_{2} K T}\left(V_{c}+R_{s} I_{c}\right)}-1\right]-\frac{\left(V_{c}+R_{s} I_{c}\right)}{R_{s h}}
\end{aligned}
$$

The reverse saturation currents of the two diodes are expressed in terms of their values at reference temperature and irradiance as (Ishaque et al., 2011; Chan and Phang, 1987; Sandrolini et al., 2010)

$$
I_{o s 1}=I_{o r 1}\left[\frac{T}{T_{r}}\right]^{3} e^{\frac{q E_{g}}{A_{1} K}\left(\frac{1}{T_{r}}-\frac{1}{T}\right)}
$$

and

$$
I_{o s 2}=I_{o r 2}\left[\frac{T}{T_{r}}\right]^{3} e^{\frac{q E_{g}}{A_{2} K}\left(\frac{1}{T_{r}}-\frac{1}{T}\right)}
$$

Equations (5), (2), (6) and (7) represent the mathematical model based on the double-diode equivalent circuit. The model parameters are series resistance $\left(R_{S}\right)$, shunt resistance $\left(R_{s h}\right)$, ideality factors for both diodes $\left(A_{1}\right.$ and $\left.A_{2}\right)$, nominal short-circuit current $\left(I_{n}\right)$, reverse saturation current at reference temperature and irradiance for both diodes $\left(I_{o r 1}\right.$ and $\left.I_{o r 2}\right)$ and short-circuit current temperature coefficient $\left(k_{i}\right)$. It is obvious that the second diode adds two parameters to the model. It should be mentioned that the manufacturer datasheet usually provides values for the nominal short-circuit current $\left(I_{n}\right)$ and short-circuit current temperature coefficient $\left(k_{i}\right)$.

Figure 1 Single-diode model of (a) PV cell and (b) PV array

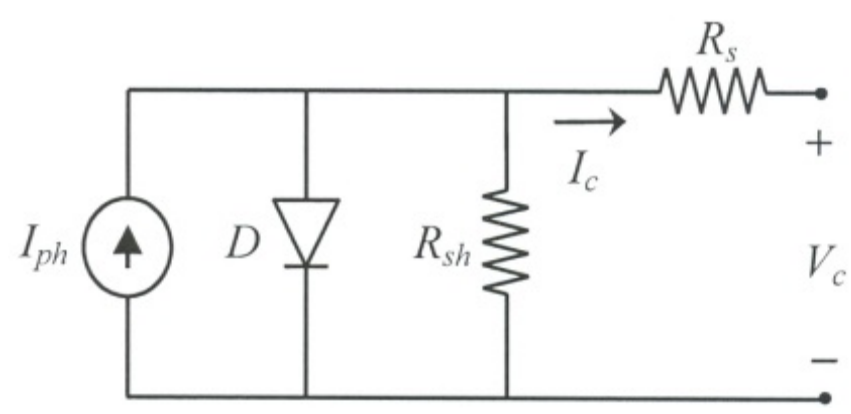

(a)

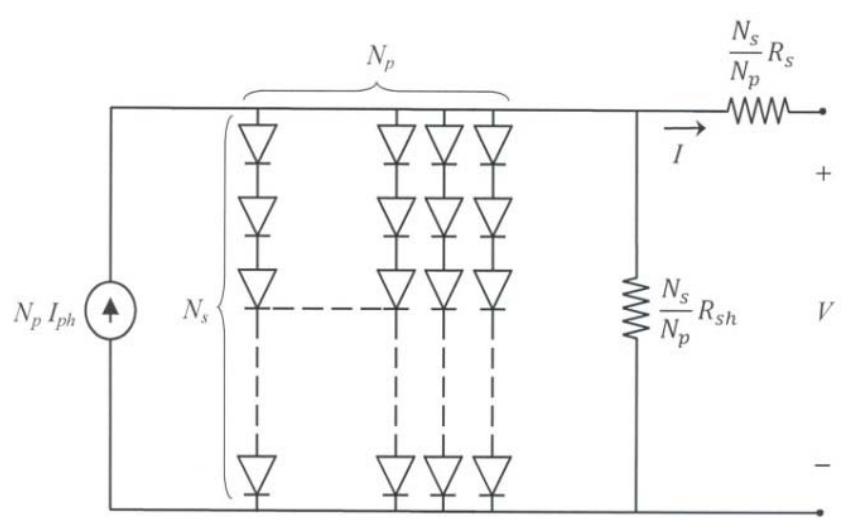

(b)
Figure 2 Double-diode model of a PV cell

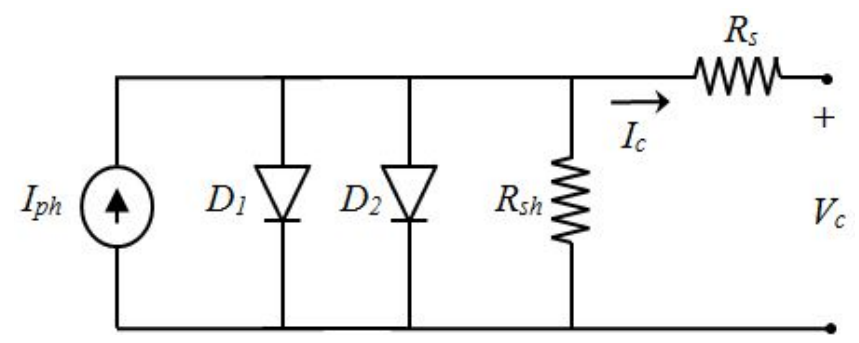

The objective of this research is to estimate the model parameters of PV modules by minimising the discrepancy between computed and targeted performance. In other words, a set of parameters, which yields computed performance as close as possible to targeted performance is sought. The targeted performance could be attained from the datasheet supplied by the manufacturer or measured in the laboratory. Targeted performance of the datasheet signifies four indices, i.e., the short-circuit current, open-circuit voltage, maximum power and voltage at maximum power, all at STC. Measurements of module output voltage and current could be also used as targeted performance. An acceptable set of parameters has to minimise the RAE that measures the discrepancy between computed and targeted performance. The RAE is expressed as

$$
R A E=\sum_{i=1}^{n} \frac{\left|X_{C i}-X_{T i}\right|}{X_{T i}}
$$

where $X_{C i}$ is the $i^{\text {th }}$ computed index, $X_{T i}$ is the $i^{\text {th }}$ targeted index and $n$ is the number of performance indices. The RAE given in equation (8) is considered an objective function to be minimised via optimisation. The problem is independently solved by GA, PSO and BF algorithms.

\section{Optimisation routines}

\subsection{Genetic algorithms}

The GA is a probabilistic random guided search technique inspired by the Darwinian theory of evolution, which employs the 'survival of the fittest' concept of natural biology (Goldberg, 1989; Deb, 2000; Deep et al., 2009). One distinct feature of GA is that the routine starts searching from a population of points, not a single point, with no need to have information about the derivatives of the objective function. The algorithm codes prospective solutions of the problem as a population of individual chromosomes composed of different genes. Each gene carries the value of one variable of the objective function. The population is randomly initialised and the individuals are evaluated based on the corresponding values of the objective function. Fit individuals are probabilistically copied to the 'mating pool', while weak individuals likely die as their probability of selection is small due to poor fitness. The natural genetic processes of crossover and mutation are then imitated in order to mate parents of the current generation, and produce the offspring of the new generation. 
Crossover means random gene exchange between selected individuals. The process is usually carried out between two individuals of the current generation (parents) to yield two different individuals of the next generation (offspring). On the other hand, mutation is the random gene alteration which is a typical phenomenon in natural biology. Mutation means that one offspring might have a genetic characteristic that is not directly found in either parent. Mutation is performed in GA at a very little probability; yet, it helps the routine escape local minima.

The routine continues till the termination criterion is satisfied, where the best individual of the last generation is taken as the solution of the problem. The termination condition could be stagnation of fitness value over certain number of generations, reaching runtime limit, obtaining desired fitness value, executing a preset number of generations or a combination of such criteria. Based on Darwin's theory, the population evolves from one generation to the next as the best fitness improves.

In spite of the remarkable robustness of GA in finding global optima, the slowness of operation could be a significant obstacle in some applications. However, GA has shown notable effectiveness in many types of optimisation problems. Coding of individual solutions has granted GA one more apparent plus, which is its adaptability to certain optimisation problems that could not be solved by classical or even some other evolutionary techniques. The sequentially applied genetic operations of selection, crossover and mutation attributed GA with the ability to escape local optima, besides the robustness of attaining global ones.

\subsection{Particle swarm optimisation}

The PSO, which is a member of the nature-inspired routines family, mimics the social behaviour of a swarm of particles (birds, insects, fish, etc.) seeking the richest food source in a large field (Kennedy and Eberhart, 1995; Eberhart and Kennedy, 1995; Mendes et al., 2004). Memories and communications between particles are employed to enhance search efficiency and robustness. The algorithm exploits a population of randomly generated potential solutions in order to detect the global minimum of a highly nonlinear multimodal objective function. The PSO is a derivative-free algorithm, which utilises cooperation, competition and experience of the swarm particles along with probabilistic transition rules of search. In summary, the natural intelligent behaviour of particle swarms is being copied accordingly to computer software in order to accomplish a complicated search mission for optimisation purposes.

The algorithm starts with a randomly generated swarm of individual particles representing potential solutions to the optimisation problem. Each particle signifies a position in the search space at which the objective function is evaluated. The particles move in the search space affected by three factors, namely, the previous movement of the particle (inertia effect), the best position visited by the particle (personal best) and the best position of the whole swarm over the previous iterations (global best). The new velocities of the particles are probabilistically determined as given in equation (9), while the new positions are computed as shown in equation (10). One distinct feature of PSO, compared to other evolutionary techniques, is that it does not employ the 'survival of the fittest' concept as it does not implement a direct selection function. In other words, particles of low fitness can survive and probably visit any point in the search space. Particle velocity and position are updated as

$$
\begin{aligned}
& v_{i}^{k+1}=w v_{i}^{k}+\alpha\left(P_{p}^{k}-x_{i}^{k}\right)+\beta\left(P_{g}^{k}-x_{i}^{k}\right) \\
& x_{i}^{k+1}=x_{i}^{k}+v_{i}^{k+1}
\end{aligned}
$$

where $v_{i}^{k+1}$ is the velocity of the $i^{\text {th }}$ particle at the $(k+1)^{t h}$ iteration, $w$ is the inertia weighting factor, $v_{i}^{k}$ is the velocity vector of the $i^{\text {th }}$ particle at the $k^{\text {th }}$ iteration, $\alpha$ and $\beta$ are bounded positive uniformly distributed random numbers, $P_{p}^{k}$ is the position vector of the personal best location at the $k^{\text {th }}$ iteration, $x_{i}^{k}$ is the position vector of the $i^{\text {th }}$ particle at the $k^{\text {th }}$ iteration, $P_{g}^{k}$ is the position vector of the global best location up to the $k^{\text {th }}$ iteration and $x_{i}^{k+1}$ is the position vector of the $i^{\text {th }}$ particle at the $(k+1)^{\text {th }}$ iteration.

One advantage of PSO is the obvious ease of implementation. Once the particle positions and velocities are randomly initialised, equations (9) and (10) are used to iterate on the objective function till convergence.

\subsection{Bacterial foraging}

The BF optimisation algorithm is based on the foraging theory of the E. coli bacteria which comprises three main activities, namely, chemotaxis, reproduction and elimination-dispersal (Passino, 2002; Tang et al., 2006). Chemotaxis indicates the motion pattern of bacteria in the presence of chemical attractants and repellants. During a chemotactic step, a bacterium can either swim or tumble, where swimming means continuous movement in some direction and tumbling signifies random change of direction. Asexual reproduction of the E. coli bacteria happens under certain conditions when a bacterium elongates then splits from the middle into two identical individuals. Elimination-dispersal probabilistically takes place due to sudden environmental changes. The bacteria may die affected by noxious substances, while new bacteria can be randomly generated at different location. While foraging, the $E$. coli bacteria have a tendency of swarming through communication by chemicals. The foraging theory is based on maximising the energy gained per unit time. A bacterium is in continuous search of nutrient-rich media using its four-second memory to compute the nutrient gradient.

A computer program, which imitates foraging activities of the $E$. coli bacteria can be used for optimising a multi-modal nonlinear function with no derivative information required. In the optimisation software, 
chemotaxis is modelled via a step taken by the bacterium in a random direction as

$$
\theta^{i}(j+1, k, l)=\theta^{i}(j, k, l)+C(i) \frac{\Delta(i)}{\sqrt{\Delta^{T}(i) \cdot \Delta(i)}}
$$

where $\theta^{i}(j+1, k, l)$ is the position of the $i^{\text {th }}$ bacterium at the $(j+1)^{\text {th }}$ chemotactic step, $k^{\text {th }}$ reproduction step and $l^{\text {th }}$ elimination-dispersal event, $\theta^{i}(j, k, l)$ is the position of the $i^{\text {th }}$ bacterium at the $j^{\text {th }}$ chemotactic step, $k^{\text {th }}$ reproduction step and $l^{\text {th }}$ elimination-dispersal event, $C(i)$ is the step length of the $i^{\text {th }}$ bacterium and $\Delta(i)$ is a random vector in the search space. The swarming effect is modelled by adding a value, representing the cell-to-cell communication, to the objective function. Such value depends on the width and depth of the attractant and repellant chemicals released by one bacterium.

$$
\begin{aligned}
J_{c c}(\theta, P(j, k, l))= & \sum_{i=1}^{S}\left[-d_{\text {attract }} e^{\left(-w_{\text {attract }} \sum_{m=1}^{p}\left(\theta_{m}-\theta_{m}^{i}\right)^{2}\right)}\right] \\
& +\sum_{i=1}^{S}\left[-h_{\text {repell }} e^{\left(-w_{\text {repell }} \sum_{m=1}^{p}\left(\theta_{m}-\theta_{m}^{i}\right)^{2}\right)}\right]
\end{aligned}
$$

where $J_{c c}(\theta, P(j, k, l))$ is the cell-to-cell effect to be added to the objective function of the bacterium $\theta, P(j, k, l)$ is the bacteria population at the $j^{\text {th }}$ chemotactic step, $k^{\text {th }}$ reproduction step and $l^{\text {th }}$ elimination-dispersal event, $S$ is the population size, $d_{\text {attract }}$ and $w_{\text {attract }}$ are the depth and width of attractant chemical, $p$ is dimension of the search space and $h_{\text {repell }}$ and $w_{\text {repell }}$ are the height and width of repellant chemical.

At each reproduction step, the bacteria population is ranked based on the associated values of objective function. Assuming the number of reproduced bacteria to be $S_{r}$, the worst $S_{r}$ bacteria are deleted from the population, whereas the best $S_{r}$ bacteria are split in half at the same position. Such reproduction scenario assures fixed population size. The elimination-dispersal event takes place when $N_{e d}$ bacteria are randomly eliminated from the population with a probability of $p_{e d}$ and an equal number of bacteria is dispersed at random positions of the search space.

The BF algorithm is known for its robustness and effectiveness of optimising highly-nonlinear multi-modal objective functions. The algorithm has been successfully applied to many engineering optimisation problems.

\section{Results and case studies}

The proposed optimisation-based technique of parameter estimation is applied to three PV modules with different ratings and manufacturers. The three case studies aim at showing a comparison of the optimisation routines, ability to model PV modules under extreme operating conditions and adaptability to nature of targeted performance and number of sought parameters. The method is implemented in MATLAB platform. The objective function is coded as an m-file to minimise the error given in equation (8) based on a comparison between performance computed from the mathematical model of equations (1) through (7) and targeted performance. PSO and $\mathrm{BF}$ algorithms are also coded in m-files, whereas the GA toolbox of MATLAB is used to run GA.

\subsection{Case study 1: comparison of optimisation routines}

In this case study, the equivalent circuit parameters of sharp ND240QCJ solar module are estimated using the GA, PSO, and BF algorithms. Parameters of both single- and double-diode models are estimated based on datasheet information. The performance of optimisation algorithms is compared on the given nonlinear problem and the modelling accuracy of obtained parameters is investigated. The electrical characteristics of the PV module (Table A1) include the open-circuit voltage, $V_{o c}$, short-circuit current, $I_{s c}$, maximum power, $P_{\max }$ and voltage at maximum power point, $V_{M P P}$, under STC. Such four indices are used as the targeted performance of the module. The datasheet also gives values of the nominal short-circuit current $\left(I_{n}\right)$ and short-circuit current temperature coefficient $\left(k_{i}\right)$. Accordingly, the missing parameters of the single-diode model are the series resistance $\left(R_{s}\right)$, shunt resistance $\left(R_{s h}\right)$, diode ideality factor $(A)$ and reverse saturation current of the diode at reference temperature and irradiance $\left(I_{o r}\right)$. In addition to these four parameters, the double-diode model misses the ideality factor and reverse saturation current of the second diode, too. In other words, the search space is of four and six dimensions in case of single- and double-diode models, respectively.

The objective function given in equation (8) is minimised independently using GA, PSO and BF; the optimisation results are given in Table 1. For fair comparison, all three algorithms are run for 300 iterations with a population of 40 individuals. The condition of 300 iterations is met with BF through 25 chemotactic steps, four reproduction steps and three elimination-dispersal events. Every algorithm is run for ten times; the best result over the ten runs is shown in Table 1 . The convergence behaviours of the three algorithms during the best run are compared in Figures 3 and 4 for parameter estimation of the single- and double-diode models, respectively.

It appears from Table 1 that for both equivalent circuit models, the best objective function value is obtained through PSO and the fastest converging algorithm is also PSO. However, over the ten runs of each algorithm on single-diode model, the average convergence time in seconds is $14.88,9.95$ and 46.89 while the average objective function is $0.0073,0.0032$ and 0.0030 for GA, PSO and BF, respectively. The ten run averages on the double-diode model are 15.47, 11.11 and 53.49 sec. for the convergence time, $\backslash$ and $0.0128,0.0040, \backslash$ and 0.0028 for the objective function obtained via GA, PSO, $\backslash$ and $\mathrm{BF}$, respectively. It seems that the average objective function of $\mathrm{BF}$ is the best, 
which indicates highest robustness. Otherwise, PSO always outperforms GA and BF on solving the current problem.

Figure 3 Convergence behaviours of the optimisation algorithms on the single-diode mode (see online version for colours)

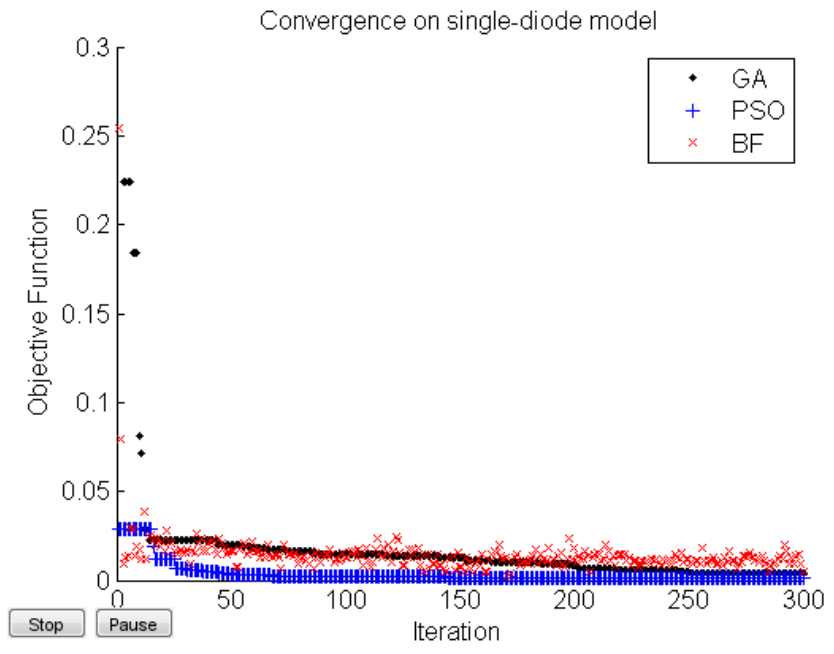

Figure 4 Convergence behaviours of the optimisation algorithms on the double-diode mode (see online version for colours)

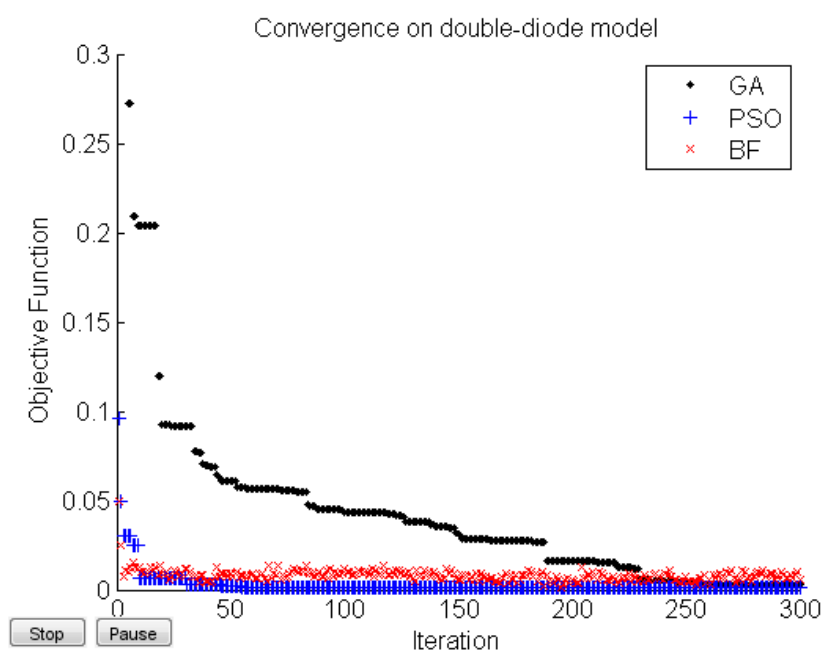

The parameter sets in Table 1 are used to compute the performance characteristics of the PV module in order to compare to targeted values. The comparison is held in Table 2, where PSO computed performance appears to be almost always better than those of GA and BF. It should be noted that the objective function value of the double-diode model is always less than that of the single-diode model, whereas the convergence time is higher. Such observation is true for all three algorithms on the best case of the ten runs, Table 1. Apparently, the double-diode model is more precise in representing module performance at STC.

The BF algorithm is distinctly slower than GA and PSO due to its heavy computational burden per iteration. However, PSO is the fastest since it has the least computational load per iteration. One other reason behind the high convergence speed of PSO is the possibility of vectorising some procedural steps over the whole population. On the contrary, computations of both GA and $\mathrm{BF}$ are to be performed over a single individual or a pair of individuals at most. One observation from Figures 3 and 4 is that the objective function value either stagnates or decreases in case of GA and PSO, while it fluctuates with $\mathrm{BF}$. The reason is that the $\mathrm{BF}$ algorithm has no inherent procedure that preserves the best objective function value while iterating. However, the global best in PSO and the elite strategy in GA maintain the best function value met so far at any iteration. Nevertheless, the iteration-wise best objective function value could be saved in BF along with its corresponding location, and the minimum could be taken as the optimisation solution.

Experimental measurements are used to validate the parameters of the PV module. A variable resistor is used to load the PV module at different voltages from open-circuit to short-circuit. Voltage and current readings are recorded under two operating conditions at $970 \mathrm{~W} / \mathrm{m}^{2}$ irradiance with $40^{\circ} \mathrm{C}$ temperature and at $800 \mathrm{~W} / \mathrm{m}^{2}$ irradiance with $48^{\circ} \mathrm{C}$ temperature. Measurements are compared to performance computed through single- and double-diode models in Figures 5 and 6, respectively. The good matching between experimental and analytical results in Figures 5 and 6, considering the various random and systematic errors associated with experimental work indicates the effectiveness of the proposed method.

Table 1 Estimated parameters of the sharp ND240QCJ module

\begin{tabular}{|c|c|c|c|c|c|c|}
\hline \multirow{2}{*}{ Parameter } & \multicolumn{3}{|c|}{ Single-diode model } & \multicolumn{3}{|c|}{ Double-diode model } \\
\hline & $G A$ & PSO & $B F$ & $G A$ & PSO & $B F$ \\
\hline Objective function & 0.0036 & 0.0017 & 0.0021 & 0.0028 & 0.0014 & 0.0017 \\
\hline Convergence time, sec & 13.82 & 9.512 & 46.316 & 14.93 & 10.753 & 50.27 \\
\hline$R_{s}, \Omega$ & 1.3487 & 1.2234 & 1.1598 & 1.0909 & 1.0225 & 1.0287 \\
\hline$R_{s h}, \Omega$ & 1037.9 & 1154 & 1386 & 797.68 & 1489.2 & 1364.4 \\
\hline$A_{1}$ & 1.0912 & 1.1259 & 1.1395 & 1.1408 & 1.176 & 1.1694 \\
\hline$I_{o r 1}, A$ & $1.849 \times 10^{-9}$ & $3.638 \times 10^{-9}$ & $4.722 \times 10^{-9}$ & $4.790 \times 10^{-9}$ & $9.134 \times 10^{-9}$ & $8.143 \times 10^{-9}$ \\
\hline$A_{2}$ & - & - & - & 1.8295 & 2.5 & 1.9436 \\
\hline$I_{o r 2}, A$ & - & - & - & $1 \times 10^{-7}$ & $1 \times 10^{-7}$ & $9.311 \times 10^{-8}$ \\
\hline
\end{tabular}


Table 2 Computed performance indices of the sharp ND240QCJ module

\begin{tabular}{|c|c|c|c|c|c|c|}
\hline \multicolumn{3}{|c|}{ Performance index (STC) } & \multirow{2}{*}{$\frac{V_{o c}, V}{37.5}$} & \multirow{2}{*}{$\begin{array}{c}I_{s c}, A \\
8.75\end{array}$} & \multirow{2}{*}{$\frac{P_{\max }, W}{240}$} & \multirow{2}{*}{$\begin{array}{c}V_{M P P}, V \\
29.3\end{array}$} \\
\hline Targeted value & & & & & & \\
\hline \multirow[t]{6}{*}{ Single-diode model } & GA & Value & 37.481 & 8.739 & 240.28 & 29.28 \\
\hline & & Error, \% & -0.051 & -0.13 & 0.117 & -0.068 \\
\hline & PSO & Value & 37.499 & 8.74 & 239.99 & 29.28 \\
\hline & & Error, \% & -0.003 & -0.114 & -0.004 & -0.068 \\
\hline & $\mathrm{BF}$ & Value & 37.495 & 8.743 & 240.1 & 29.28 \\
\hline & & Error, \% & -0.013 & -0.08 & 0.042 & -0.068 \\
\hline \multirow[t]{6}{*}{ Double-diode model } & GA & Value & 37.497 & 8.738 & 239.99 & 29.34 \\
\hline & & Error, \% & -0.008 & -0.137 & -0.004 & 0.137 \\
\hline & PSO & Value & 37.499 & 8.744 & 239.99 & 29.28 \\
\hline & & Error, \% & -0.003 & -0.069 & -0.004 & -0.068 \\
\hline & $\mathrm{BF}$ & Value & 37.491 & 8.743 & 239.98 & 29.28 \\
\hline & & Error, \% & -0.024 & -0.08 & -0.008 & -0.068 \\
\hline
\end{tabular}

Figure 5 Comparison of experimental measurements and computed performance of the single-diode model of sharp ND240QCJ module (see online version for colours)

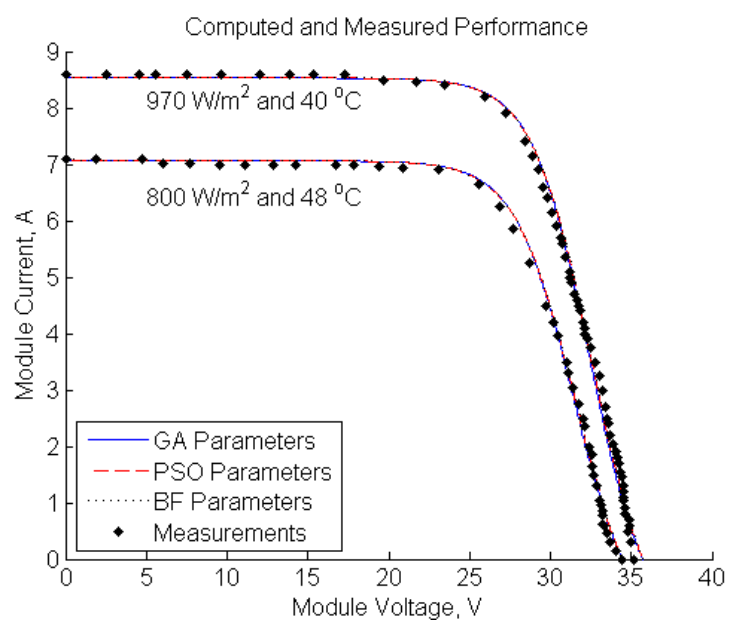

Figure 6 Comparison of experimental measurements and computed performance of the double-diode model of sharp ND240QCJ module (see online version for colours)

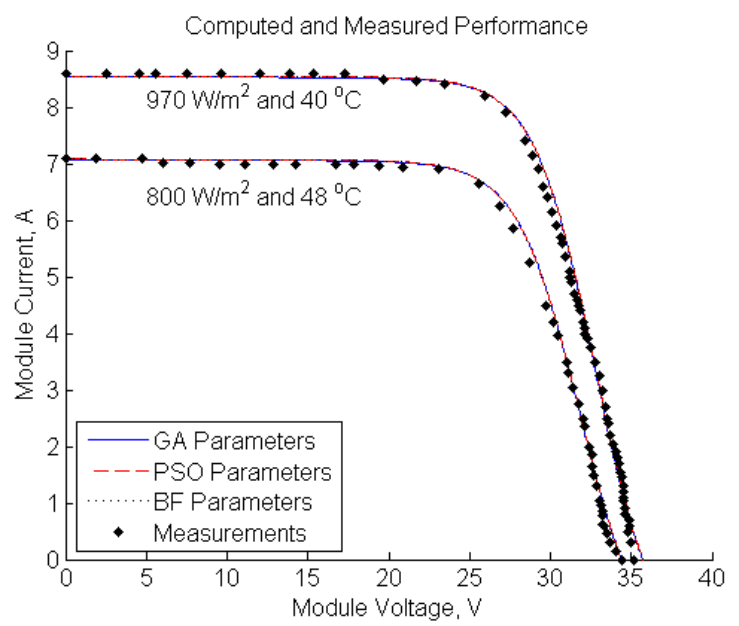

\subsection{Case study 2: modelling of extreme conditions}

The objective of this case study is to show the suitability of parameters extracted by the proposed method to model the performance of PV modules under the extreme conditions of low irradiance and partial shading. Parameters of the single- and double-diode models of an Eclipsall ME2-72P module are estimated via PSO since it showed superiority to both $\mathrm{GA}$ and $\mathrm{BF}$ in the previous case study. Information of the manufacturer datasheet, given in Table A1 is used to find the module parameters. The four indices of $V_{o c}, I_{s c}, P_{\max }$ and $V_{M P P}$ at STC are used as the targeted performance of the module. It should be noted that partial shading was modelled in the laboratory by covering selected cells by different layers of translucent sheets of paper which tend to block light rays falling on the covered cell. The pyranometer sensor is always located under the covering paper in order to record the actual irradiance hitting the cell. Different levels of irradiance on various numbers of cells can be accordingly created.

The algorithm is run for 300 iterations with swarm size of 40 particles for single- and double-diode models. PSO is run ten times for each model and the best result is taken as the solution of the optimisation problem. The run time of the single-diode model is always less than that of the double-diode model due to the lesser computational burden per iteration. The obtained parameters are listed in Table 3.

Computed and measured performances are compared at low irradiance and partial shading conditions as shown in Figure 7. At the presence of bypass diodes, the model in Patel and Agarwal (2008) is used to simulate module characteristics under the partial shading condition when 24 cells receive $147 \mathrm{~W} / \mathrm{m}^{2}, 24$ cells receive $74 \mathrm{~W} / \mathrm{m}^{2}$ and 24 cells receive $48 \mathrm{~W} / \mathrm{m}^{2}$ at $38^{\circ} \mathrm{C}$ temperature. A good matching is noticed between experimental and analytical results. Therefore, the effectiveness of the proposed technique to find a set of parameters accurately representing the performance under extreme operating conditions is evident. 
Table 3 Estimated parameters of the Eclipsall ME2-72P module

\begin{tabular}{lcc}
\hline Parameter & $\begin{array}{c}\text { Single-diode } \\
\text { model }\end{array}$ & $\begin{array}{c}\text { Double-diode } \\
\text { model }\end{array}$ \\
\hline Objective function & 0.0029 & 0.0033 \\
Convergence time, sec & 10.65 & 13.46 \\
$R_{s}, \Omega$ & 0.9274 & 0.873 \\
$R_{s h}, \Omega$ & 1500 & 1500 \\
$A_{1}$ & 0.9468 & 0.9606 \\
$I_{o r 1}, A$ & $5.659 \times 10^{-11}$ & $8.204 \times 10^{-11}$ \\
$A_{2}$ & - & 2.3154 \\
$I_{o r 2}, \mathrm{~A}$ & - & $9.958 \times 10^{-8}$ \\
\hline
\end{tabular}

Figure 7 Comparison of experimental measurements and computed performance of the Eclipsall ME2-72P module (see online version for colours)

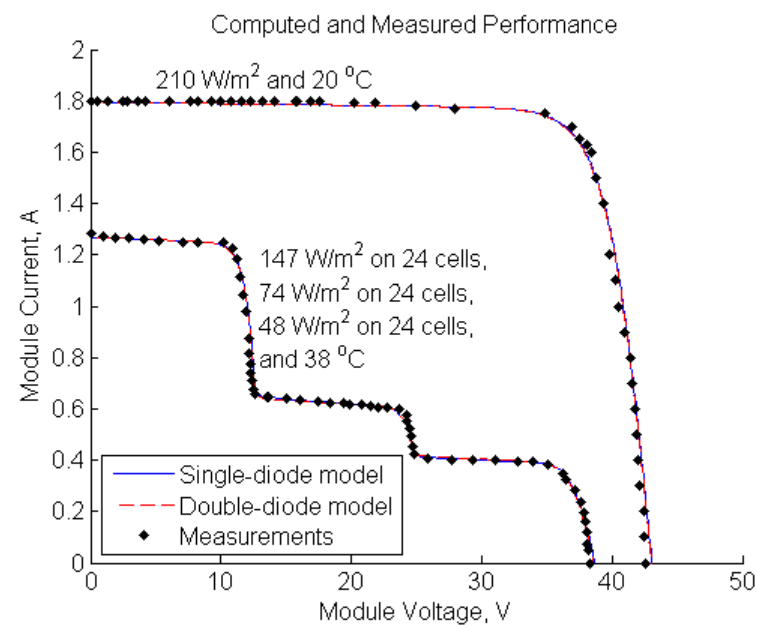

\subsection{Case study 3: parameters extracted from measurements}

The targeted performance used with the objective function in equation (8) could be obtained through measurements in case datasheet is not available or in case the module performance is deteriorated due to aging. The nominal short-circuit current at STC $\left(I_{n}\right)$ and short-circuit current temperature coefficient $\left(k_{i}\right)$ are assumed unknown. Therefore, the search space of single- and double-diode models accordingly becomes of dimension 6 and 8 , respectively. Measurements are taken in the laboratory on an LDK C1D2-140P solar module. The open-circuit voltage and short-circuit current at three different conditions are recorded. Such voltage and current readings shown in Table 4 are considered the targeted performance of the objective function.

Comparing the six targeted measurements to their corresponding computed values, the error function in equation (8) is minimised using PSO. The algorithm is run for 300 iterations with swarm size of 60 and 80 particles for the single- and double-diode models, respectively. The best results of ten runs on each model are given in Table 5. To validate the obtained parameters, experimental measurements of module performance are compared to analytical results at two different operating conditions, Figure 8 . Measurements of module voltage and current are taken at $875 \mathrm{~W} / \mathrm{m}^{2}$ irradiance with $23^{\circ} \mathrm{C}$ temperature and at $84 \mathrm{~W} / \mathrm{m}^{2}$ irradiance with $21{ }^{\circ} \mathrm{C}$ temperature. The methodology is validated by the good matching between measured and computed performance of Figure 8, even at low irradiance operation. On all cases reported in this paper, computed performances of the single- and double-diode models are very close to each other.

Table 4 Measured performance of the LDK C1D2-140P module

\begin{tabular}{llll}
\hline Operating point & 1 & 2 & 3 \\
Irradiance, $\mathrm{W} / \mathrm{m}^{2}$ & 95 & 165 & 850 \\
Temperature, ${ }^{\circ} \mathrm{C}$ & 20 & 19 & 23 \\
Open-circuit voltage, $\mathrm{V}$ & 20.5 & 21.05 & 22.35 \\
Short-circuit current, A & 0.8 & 1.3 & 7.1 \\
\hline
\end{tabular}

Figure 8 Comparison of experimental measurements and computed performance of the LDK C1D2-140P module (see online version for colours)

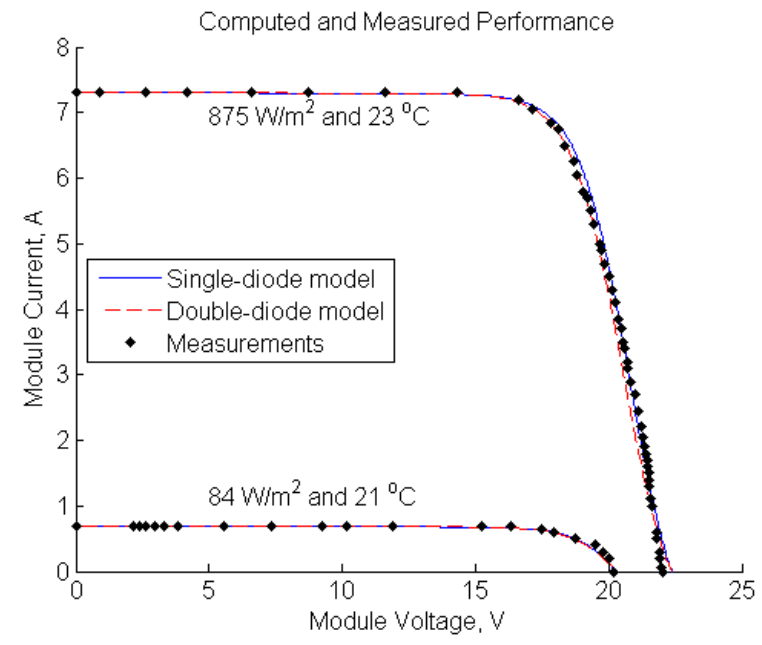

Table 5 Estimated parameters of the LDK C1D2-140P module

\begin{tabular}{lcc}
\hline Parameter & $\begin{array}{c}\text { Single-diode } \\
\text { model }\end{array}$ & $\begin{array}{c}\text { Double-diode } \\
\text { model }\end{array}$ \\
Objective function & 0.0693 & 0.0692 \\
Convergence time, sec & 27.12 & 44.58 \\
$R_{s}, \Omega$ & 0.7257 & 0.962 \\
$R_{s h}, \Omega$ & 647.31 & 756.15 \\
$A_{1}$ & 1.0136 & 1.0152 \\
$I_{o r 1}, A$ & $3.661 \times 10^{-10}$ & $3.801 \times 10^{-10}$ \\
$A_{2}$ & - & 2.1843 \\
$I_{o r 2}, A$ & - & $9.981 \times 10^{-8}$ \\
$I_{n}, A$ & 8.3791 & 8.3803 \\
$k_{i}, \% /{ }^{\circ} \mathrm{C}$ & 0.033344 & 0.046402 \\
\hline
\end{tabular}




\section{Conclusions}

The paper presents a technique for parameter estimation of PV modules based on global search and optimisation algorithms. A set of equivalent circuit parameters which minimises the error between targeted and computed performance indices of the PV module is searched. Targeted performance could be obtained from the manufacturer datasheet or measured in the laboratory. The technique could be arbitrarily applied to single- or double-diode models.

Three case studies of different objectives are reported in this paper. In the first case study, the global optimisation algorithms of GA, PSO and BF are used to extract PV module parameters of the single- and double-diode models from datasheet information. Comparing the performance of all three algorithms for the same population size and number of iterations, it is shown that PSO gives best results in shortest convergence time. Otherwise, $\mathrm{BF}$ is characterised by the highest robustness.

The parameters of a different PV module are estimated via datasheet information using PSO in the second case study. The module performance is modelled under the extreme operating conditions of low irradiance and partial shading. The good matching between measured and computed performance indicates the accuracy of modelling.

The third case study shows the flexibility of the proposed method regarding the source of targeted performance and number of estimated parameters. Assuming datasheet is missing, measured open-circuit voltages and short-circuit currents of another PV module is used as targeted performance. Meanwhile, the number of sought parameters accordingly increases. A good matching between experimental and analytical performances of the module is also observed.

One distinct advantage of the proposed methodology, in comparison to other techniques available in the literature, is the usage of a simple and straightforward formulation of the objective function to be minimised. It is also flexible on the nature of the PV model, source of targeted performance, number of sought parameters and global optimisation algorithm to be used.

\section{References}

Ahmad, M., Talukder, A. and Tanni, M. (2012) 'Estimation of important parameters of photovoltaic modules from manufacturer's datasheet', Proc. IEEE Int. Conf. Informatics, Electronics, and Vision, Dhaka, Bangladesh, 18-19 May, pp.571-576.

Awadallah, M. A. (2016) 'Variations of the bacterial foraging algorithm for the extraction of PV module parameters from nameplate data', Energy Conversion and Management, Vol. 113, pp.312-320.

Awadallah, M.A. and Venkatesh, B. (2016) 'Bacterial foraging algorithm guided by particle swarm optimization for parameter identification of PV modules', IEEE Canadian Journal of Electrical and Computer Engineering, Vol. 39, No. 2, pp.150-157.
Bouzidi, K., Chegaar, M. and Bouhemadou, A. (2007) 'Solar cells parameters evaluation considering the series and shunt resistance', Solar Energy Materials and Solar Cells, Vol. 91, No. 18 , pp.1647-1651.

Chan, D. and Phang, J. (1987) 'Analytical methods for the extraction of solar-cell single- and double-diode model parameters from I-V characteristics', IEEE Trans. Electron Devices, Vol. 34, No. 2, pp.286-293.

Chan, D., Phillips, J. and Phang, J. (1986) 'A comparative study of extraction methods for solar cell model parameters', Solid-State Electronics, Vol. 29, No. 3, pp.329-337.

Chatterjee, A., Keyhani, A. and Kapoor, D. (2011) 'Identification of photovoltaic source models', IEEE Trans. Energy Conversion, Vol. 26, No. 3, pp.883-889.

Chegaar, M., Azzouzi, G. and Mialhe, P. (2006) 'Simple parameter extraction method for illuminated solar cells', Solid-State Electronics, Vol. 50, No. 7, pp.1234-1237.

Chenni, R., Makhlouf, M., Kerbache, T. and Bouzid, A. (2007) 'A detailed modeling method for photovoltaic cells', Energy, Vol. 32, No. 9, pp.1724-1730.

Chin, V.J., Salam, Z. and Ishaque, K. (2017) 'An accurate and fast computational algorithm for the two-diode model of PV module based on a hybrid method', IEEE Trans. Industrial Electronics, Vol. 64, No. 8, pp.6212-6222.

Cubas, J., Pindado, S. and Farrahi, A. (2013) 'New method for analytical photovoltaic parameter extraction', Proc. Int. Conf. Renewable Energy Research and Applications, Madrid, Spain, 20-23 October, pp.873-877.

da Costa, W., Fardin, J., Simonetti, D. and Neto, L. (2010) 'Identification of photovoltaic model parameters by differential evolution', Proc. IEEE Int. Conf. Industrial Technology, Vina del Mar, Chile, 14-17 March, pp.931-936.

de Blas, M., Torres, J., Prieto, E. and Garcia, A. (2002) 'Selecting a suitable model for characterizing photovoltaic devices', Renewable Energy, Vol. 25, No. 3, pp.371-380.

Deb, K. (2000) 'An efficient constraint handling method for genetic algorithms', Computer Methods in Applied Mechanics and Engineering, Vol. 186, Nos. 2-4, pp.311-338.

Deep, K., Singh, K., Kansal, M. and Mohan, C. (2009) 'A real coded genetic algorithm for solving integer and mixed integer optimization problems', Applied Mathematics and Computation, Vol. 212, No. 2, pp.505-518.

Eberhart, R. and Kennedy, J. (1995) 'A new optimizer using particle swarm theory', Proc. Int. Symposium Micro Machine and Human Science, Nagoya, Japan, 4-6 October, pp.39-43.

Farivar, G. and Asaei, B. (2011) 'A new approach for solar module temperature estimation using simple diode model', IEEE Trans. Energy Conversion, Vol. 26, No. 4, pp.1118-1126.

Goldberg, D. (1989) Genetic Algorithms in Search, Optimization, and Machine Learning, Addison-Wesley, Reading, MA, USA.

Haouari-Merbah, M., Belhamel, M., Tobias, I. and Ruiz, J. (2005) 'Extraction and analysis of solar cell parameters from the illuminated current-voltage curve', Solar Energy Materials and Solar Cells, Vol. 87, Nos. 1-4, pp.225-233.

Hejri, M., Mokhtari, H., Azizian, M.R. and Soder, L. (2016) 'An analytical-numerical approach for parameter determination of a five-parameter single-diode model of photovoltaic cells and modules,' International Journal of Sustainable Energy, Vol. 35, No. 4, pp.396-410. 
Ikegami, T., Maezono, T., Nakanishi, F. et al. (2001) 'Estimation of equivalent circuit parameters of PV module and its application to optimal operation of PV system', Solar Energy Materials and Solar Cells, Vol. 67, Nos. 1-4, pp.389-395.

Ishaque, K. and Salam, Z. (2011) 'An improved modeling method to determine the model parameters of photovoltaic (PV) modules using differential evolution (DE)', Solar Energy, Vol. 85, No. 9, pp.2349-2359.

Ishaque, K., Salam, Z. and Syafaruddin (2011) 'A comprehensive MATLAB simulink PV system simulator with partial shading capability based on two-diode model', Solar Energy, Vol. 85, No. 9, pp.2217-2227.

Jia, Q., Ebihara, K. and Ikegami, T. (1995) 'Analytical solution for solar cell model parameters from illuminated current-voltage characteristics', Philosophical Magazine B, Vol. 7, No. 4, pp.375-382.

Kennedy, J. and Eberhart, R. (1995) 'Particle swarm optimization', Proc. IEEE Int. Conf. Neural Networks, Perth, Australia, 27 November-1 December, Vol. 4, pp.1942-1948.

Krishnakumar, N., Venugopalan, N. and Rajasekar, N. (2013) 'Bacterial foraging algorithm based parameter estimation of solar PV model', Proc. Int. Conf. Microelectronics, Communication, and Renewable Energy, Kanjirapally, Kerala, India, 4-6 June, pp.1-6.

Lo Brano, V., Orioli, A., Ciulla, G. and Di Gangi, A. (2010) 'An improved five-parameter model for photovoltaic modules', Solar Energy Materials and Solar Cells, Vol. 94 No. 8, pp.1358-1370.

Maoucha, A., Djeffal, F., Arar, D. et al. (2012) 'An accurate organic solar cell parameters extraction approach based on the illuminated I-V characteristics for double diode modeling', Proc. Int. Conf. Renewable Energies and Vehicular Technology, Hammamet, Tunisia, 26-28 March, pp.74-77.

Mendes, R., Kennedy, J. and Neves, J. (2004) 'The fully informed particle swarm: simpler, maybe better', IEEE Trans. Evolutionary Computation, Vol. 8, No. 3, pp.204-210.

Oliva, D., Aziz, M.A.E. and Hassanien, A.E. (2017) 'Parameter estimation of photovoltaic cells using an improved chaotic whale optimization algorithm', Applied Energy, Vol. 200, pp.141-154.

Ortiz-Conde, A., Sanchez, F. and Muci, J. (2006) 'New method to extract the model parameters of solar cells from the explicit analytic solutions of their I-V characteristics', Solar Energy Materials and Solar Cells, Vol. 90, No. 3, pp.352-361.

Passino, K. (2002) 'Biomimicry of bacterial foraging for distributed optimization and control', IEEE Control Systems Magazine, Vol. 22, No. 3, pp.52-67.

Patel, H. and Agarwal, V. (2008) 'MATLAB-based modeling to study the effects of partial shading on PV array characteristics', IEEE Trans. Energy Conversion, Vol. 23, No. 1, pp.302-310.

Phang, J., Chan, D. and Phillips, J. (1984) 'Accurate analytical method for the extraction of solar cell model parameters', Electronics Letters, Vol. 20, No. 10, pp.406-408.
Salem, F. and Awadallah, M.A. (2014) 'Parameters estimation of photovoltaic modules: comparison of ANFIS and ANN', International Journal of Industrial Electronics and Drives, Vol. 1, No. 2, pp.121-129.

Sandrolini, L., Artioli, M. and Reggiani, U. (2010) 'Numerical method for the extraction of photovoltaic module double-diode model parameters through cluster analysis', Applied Energy, Vol. 87, No. 2, pp.442-451.

Sera, D., Teodorescu, R. and Rodrigues, P. (2007) 'PV panel model based on datasheet values', Proc. IEEE Int. Symposium on Industrial Electronics, Vigo, Spain, 4-7 June, pp.2392-2396.

Siddique, H., Xu, P. and De Doncker, R. (2013) 'Parameter extraction algorithm for one-diode model of PV panels based on datasheet values', Proc. Int. Conf. Clean Electrical Power, Alghero, Sardinia, Italy, 11-13 June, pp.7-13.

Tang, W., Wu, Q. and Saunders, J. (2006) 'Bacterial foraging algorithm for dynamic environments', Proc. IEEE Congress Evolutionary Computation, Vancouver, Canada, 16-21 July, pp.1324-1330.

Ye, M., Wang, X. and Xu, Y. (2009) 'Parameter extraction of solar cells using particle swarm optimization', Journal of Applied Physics, Vol. 105, 094502.

Yeh, W.C., Lin, P. and Huang, C.L. (2017) 'Simplified swarm optimization for the solar cell models parameter estimation problem', IET Renewable Power Generation, Vol. 11, No. 8, pp.1166-1173.

Zagrouba, M., Sellami, A., Bouaicha, M. and Ksouri, M. (2010) 'Identification of PV solar cells and modules parameters using the genetic algorithms: application to maximum power extraction', Solar Energy, Vol. 84, No. 5, pp.860-866.

\section{Appendix}

Table A1 Datasheet information of the PV modules

\begin{tabular}{lcc}
\hline Make & Sharp & Eclipsall \\
\hline Model & ND240QCJ & ME2-72P \\
$V_{o c}, \mathrm{~V}$ & 37.5 & 45.1 \\
$I_{s c}, \mathrm{~A}$ & 8.75 & 8.58 \\
$P_{\max }, \mathrm{W}$ & 240 & 300 \\
$V_{M P P}, \mathrm{~V}$ & 29.3 & 36.71 \\
$k_{i}, \% /{ }^{\circ} \mathrm{C}$ & 0.053 & 0.053 \\
Number of cells & 60 & 72 \\
\hline
\end{tabular}

